\title{
BIOCARVÃO: REALIDADE E POTENCIAL DE USO NO MEIO FLORESTAL
}

\author{
BIOCHAR: REALITY AND POTENTIAL USE IN FORESTRY \\ Paulo André Trazzi ${ }^{1}$ Antonio Rioyei Higa ${ }^{2}$ Jeferson Dieckow ${ }^{3}$ \\ Antonio Salvio Mangrich ${ }^{4}$ Rosana Clara Vitoria Higa ${ }^{5}$
}

\begin{abstract}
RESUMO
O uso de resíduos vegetais carbonizados vem sendo resgatado e avaliado como alternativa para melhorar a qualidade do solo. O material gerado, denominado biocarvão, é o produto formado a partir da pirólise, que é a alteração térmica da biomassa em ambiente fechado, com suprimento limitado de oxigênio e em temperaturas relativamente baixas. A composição química e estrutural do biocarvão é altamente heterogênea, mas o pH é normalmente maior que sete. Algumas propriedades estão presentes em todos os biocarvões, incluindo alto teor de carbono e grau de aromaticidade, o que explica seu alto nível de recalcitrância. Contudo, a exata composição química e estrutural é dependente da combinação da matéria-prima e das condições de pirólise. Quando aplicado ao solo, o biocarvão pode aumentar o $\mathrm{pH}$, a capacidade de troca de cátions, $\mathrm{o}$ teor de carbono orgânico e a disponibilidade de nutrientes; alterar a abundância e funcionamento de fungos micorrízicos e prover refúgio para microrganismos nos microporos do biocarvão; e melhorar a estrutura do solo e disponibilidade de água. Todas estas características de interação com o solo fazem com que a sua utilização no meio agrícola apresente normalmente efeitos positivos para o crescimento vegetal. Acredita-se também que a utilização de biocarvão possa contribuir para o sequestro de carbono, sendo considerado por muitos como "carbono negativo", devido a sua capacidade de promover o crescimento vegetal e pela sua estabilidade no solo. Estudos com o biocarvão já atingiram proporções de escala mundial envolvendo diversas áreas e têm crescido muito nos últimos anos. No entanto, ainda existem muitas incertezas sobre a sua utilização na agricultura, devido principalmente ao fato de que os trabalhos publicados têm dado mais atenção a sua capacidade de manutenção e melhoria da fertilidade do solo e aumento da produtividade agrícola do que esclarecimentos dos possíveis riscos envolvidos na utilização do biocarvão.
\end{abstract}

Palavras-chave: crescimento vegetal; carbono pirogênico; fertilidade do solo.

\begin{abstract}
The use of carbonized plant biomass has been recovered and evaluated as an alternative of improving soil fertility. The material generated, called biochar, is the product formed by pyrolysis, which is the thermal decomposition of biomass in a closed container under limited supply of oxygen and at relatively low temperatures. The chemical and structural composition of biochar is highly heterogeneous, with the exception of $\mathrm{pH}$, which is usually higher than seven. Some properties are present in all kinds of biochar, including high carbon content and degree of aromaticity, which explains its high level of recalcitrance.

1 Engenheiro Florestal, Dr., bolsista CNPq de Pós-Doutorado, Programa de Pós-Graduação em Ciências Florestais, Universidade Federal do Espírito Santo, Av. Governador Lindemberg, 316, CEP 29550-000, Jerônimo Monteiro, Brasil.patrazzi@gmail.com.

2 Engenheiro Florestal, Dr., Professor do Departamento de Ciências Florestais, Universidade Federal do Paraná, Av. Pref. Lothário Meissner, 900, Jardim Botânico, CEP 80210-170, Curitiba (PR), Brasil. antonio.higa@gmail.com

3 Engenheiro Agrônomo, Dr., Professor do Departamento de Solos e Engenharia Agrícola, Universidade Federal do Paraná, Rua dos funcionários, 1540, Juvevê, CEP 80035-050, Curitiba (PR), Brasil. jefersondieckow@ufpr.br

4 Químico, Dr., Professor do Departamento de Química, Universidade Federal do Paraná, Centro Politécnico, Jardim das Américas, Caixa Postal 19032, CEP 81531-980, Curitiba (PR), Brasil. asmangrich@gmail.com

5 Engenheira Agrônoma, Dr ${ }^{\mathrm{a}}$., Pesquisadora do Centro Nacional de Pesquisa de Florestas, Embrapa Florestas, Estrada da Ribeira, Km 111, Guaraituba, Caixa Postal 319, CEP 83411-000, Colombo (PR), Brasil. rosana.higa@ embrapa.br
\end{abstract}

Recebido para publicação em 17/08/2015 e aceito em 23/03/2017

Ci. Fl., v. 28, n. 2, abr. - jun., 2018 
However, its exact chemical composition and structure depends on the combination of raw material and pyrolysis conditions. When applied to soil, biochar can increase $\mathrm{pH}$, cation exchange capacity and organic carbon and nutrient availability, raises in the abundance and functioning of mycorrhizal fungi, providing refuge for micro-organisms in the biochar micropores and improving soil structure and water availability. All these features in the soil interaction make its use in the agriculture normally to have positive effects on plant growth. It is believed that the use of biochar can contribute to carbon sequestration, it is considered by many as "carbon negative" due to its ability to promote plant growth and its stability in the soil. Studies with biochar have reached worldwide proportions involving different areas, and have grown a lot in recent years. However, there are still many uncertainties about its use in agriculture, mainly because the published papers have given more focus to biochar ability to maintain and improve soil fertility and increase agricultural productivity than to clarify the possible risks involved in biochar utilization.

Keywords: plant growth; pyrogenic carbon; soil fertility.

\section{INTRODUÇÃO}

O uso de resíduos orgânicos carbonizados vem sendo resgatado e avaliado como alternativa para melhorar a qualidade do solo. Através da decomposição térmica do material orgânico em condições limitadas de suprimento de oxigênio, e em temperaturas relativamente baixas, o material produzido, conhecido como biocarvão, contribui para a manutenção da qualidade do solo (LEHMANN; JOSEPH, 2009). O processo de formação do biocarvão promove um rearranjo dos átomos de carbono, aumentando a porosidade do material, e isto proporciona melhoria nas propriedades químicas, principalmente pela alta superfície específica resultando em aumento da capacidade de troca de cátions e adsorção de nutrientes (ATKINSON et al., 2010).

O biocarvão está normalmente relacionado aos solos de origem antropogênica, devido ao processo de formação ser semelhante, por meio da decomposição térmica do material vegetal. No entanto, solos antropogênicos são formados ao longo de milênios, e por isso, existe uma reposição contínua da matéria orgânica no solo. Geralmente, pesquisadores fazem a associação do biocarvão com a "Terra Preta de Índio", que é um solo altamente fértil, devido principalmente à alta capacidade de troca de cátions, aos altos teores de matéria orgânica e nutrientes como nitrogênio, fósforo, cálcio e potássio. A "Terra Preta de Índio" ocorre em pequenas porções na Região Amazônica e foi formada através da decomposição de restos de plantas e animais, em locais onde índios nômades habitavam (GLASER et al., 2001; NOVOTNY et al., 2009).

Quando aplicado ao solo, o biocarvão pode proporcionar aumento do pH, CTC e carbono orgânico (LIANG et al., 2006; CHAN et al., 2007; RONDON et al., 2007); aumento na fixação biológica de nitrogênio e na disponibilidade de boro, molibdênio, cálcio, potássio e fósforo (RONDON et al., 2007; KOOKANA et al., 2011); aumento na adsorção de herbicidas na superfície do biocarvão, diminuindo as perdas por lixiviação (SPOKAS et al., 2009); alteração na abundância e funcionamento de fungos micorrízicos, principalmente pela alteração das propriedades físicas e químicas (WARNOCK et al., 2007); provimento de refúgio para a microbiota nos microporos do biocarvão (WARNOCK et al., 2007; THIES; RILLIG, 2009); aumento na disponibilidade de nutrientes pela alteração da biota do solo (LEHMANN et al., 2011); e melhoria na estrutura do solo e disponibilidade de água (DOWNIE et al., 2009).

A utilização do biocarvão tem crescido bastante no meio agrícola, no entanto, apenas em escala experimental. A realidade para o uso do biocarvão para a produção de florestas está ainda mais distante. Poucos são os trabalhos publicados na área e, do ponto de vista prático, ainda muito tem que ser estudado. Assim, o objetivo deste trabalho é fazer uma abordagem do atual uso do biocarvão, apresentando os principais processos de produção e características, interação do biocarvão no solo e a sua influência na produtividade agrícola, evidenciando o potencial do biocarvão para a sua utilização no meio florestal.

\section{PRODUÇÃO E CARACTERÍSTICAS FÍSICO-QUÍMICAS DO BIOCARVÃO}

O biocarvão é um produto obtido por muitos processos e sua qualidade é dependente de cada processo e do material em que o processo é aplicado (SOHI et al., 2010). Por meio da pirólise, que é 
a decomposição térmica de materiais orgânicos pelo aquecimento na ausência de oxigênio, são gerados três produtos: um líquido, que é comumente chamado de bio-óleo; um material sólido, que pode ser usado como insumo no solo (biocarvão), ou então usado para geração de energia (carvão); e um produto gasoso não condensável, contendo monóxido de carbono, dióxido de carbono, hidrogênio, metano e outros hidrocarbonetos (MCCARL et al., 2009).

$\mathrm{Na}$ produção de biocarvão, existem três principais processos de degradação térmica, que são governados principalmente pela temperatura de pirólise, pelo tempo que o material permanece no reator e pela taxa de aquecimento (rampa de aquecimento). Pirólise lenta é a conversão térmica da biomassa por aquecimento em baixas e médias temperaturas, na ausência de oxigênio. Pirólise rápida é o aquecimento muito rápido da biomassa, resultando em uma maior proporção de bio-óleo e menor de biocarvão. E a gaseificação é a pirólise em altas temperaturas e tem o biocarvão como subproduto (BROWN, 2009; BROWNSORT, 2009; MCCARL et al., 2009). As condições e o rendimento da produção de biocarvão e os produtos gerados no fim do processo são altamente dependentes do tipo de pirólise utilizada (Tabela 1).

TABELA 1: Rendimento de produto por métodos de pirólise.

TABLE 1: Product yield by pyrolysis methods.

\begin{tabular}{cccccc}
\hline \multirow{2}{*}{$\begin{array}{c}\text { Tipo de } \\
\text { Pirólise }\end{array}$} & \multicolumn{2}{c}{ Condições } & \multicolumn{3}{c}{ Rendimento (\%) } \\
\cline { 2 - 6 } & Temperatura $\left(\mathrm{C}^{\circ}\right)$ & Permanência & Biocarvão & Bio-óleo & Gases \\
\hline Lenta & $500(250-750)$ & $5-30 \mathrm{~min}(\mathrm{~min}-$ dias $)$ & $35(2-60)$ & $30(0-60)$ & $35(0-60)$ \\
Intermediária & $500(320-500)$ & $10-20 \mathrm{seg}(\mathrm{min})$ & $20(19-73)$ & $50(18-60)$ & $30(9-32)$ \\
Rápida & $500(400-750)$ & $1 \mathrm{seg}(1-5 \mathrm{seg})$ & $12(0-50)$ & $75(10-80)$ & $13(10-30)$ \\
\hline Gaseificação & $>750$ & $10-20 \mathrm{seg}$ & 10 & 5 & 85 \\
\hline
\end{tabular}

As informações entre parênteses referem-se à amplitude das observações dos processos mencionados. Brown (2009), Brownsort (2009).

A composição química e estrutural do biocarvão é altamente heterogênea. Algumas propriedades estão presentes em todos os biocarvões, incluindo alto teor de $\mathrm{C}$ e grau de aromaticidade, o que explica seu alto nível de recalcitrância. Contudo, a exata composição química e estrutural é dependente da combinação da matéria-prima e das condições de pirólise. As características químicas e físicas do biocarvão podem alterar significativamente as propriedades físicas e processos do solo e são, portanto, importantes para consideração antes da aplicação no solo (VERHEIJEN et al., 2009).

Uma característica que é comum ao biocarvão é que ele compreende principalmente as formas aromáticas estáveis do carbono orgânico, e comparado ao carbono da matéria-prima que o originou, não pode ser prontamente retornado à atmosfera como gás carbônico, até mesmo em condições ambientais e biológicas favoráveis, tal como aquelas que prevalecem no solo. A temperatura de pirólise é o mais importante parâmetro a se considerar no processo de produção do biocarvão. $\mathrm{O}$ teor de $\mathrm{C}$ fixo do biocarvão está diretamente relacionado ao aumento da temperatura final de pirólise. O mesmo acontece com o teor de cinzas, ou seja, para uma mesma matéria-prima, quanto maior a temperatura de pirólise maior é o teor de cinzas produzido. Enquanto que o rendimento da produção de biocarvão é inversamente proporcional à temperatura de pirólise (SOHI et al., 2010).

Pelo fato de biocarvões apresentarem uma variedade de componentes orgânicos e inorgânicos, eles podem ser usados para melhorar a qualidade do solo. No entanto, suas propriedades físico-químicas são determinantes para o sucesso de sua utilização no solo. A variação das propriedades físico-químicas é dependente principalmente da composição elementar da matéria-prima, do processo de produção e do tamanho das partículas (NOVAK et al., 2014).

As principais características para avaliação do biocarvão utilizadas por pesquisadores são: $\mathrm{pH}$, conteúdo de materiais voláteis, teor de cinzas, capacidade de retenção de água, densidade aparente, volume de poros e superfície específica. As relações atômicas de $\mathrm{O} / \mathrm{C}, \mathrm{O} / \mathrm{H}$ e C/H também são avaliadas por fornecer uma medida confiável da extensão da pirólise e da alteração do nível oxidativo do biocarvão no solo, e são relativamente de simples determinação (SOHI et al., 2009). 


\section{BIOCARVÃO E A SUA INTERAÇÃO COM O SOLO}

As interações entre matéria orgânica e a superfície coloidal das argilas são dependentes da distribuição dos grupos funcionais da matéria orgânica, da polaridade dos componentes e da composição e concentração dos cátions e ânions na solução do solo. Reações complexas similares estão propensas a ter lugar nas superfícies dos biocarvões, principalmente se estes biocarvões apresentam alto conteúdo mineral (JOSEPH et al., 2010).

O biocarvão é bastante estável no solo e tem potencial de aumentar diretamente a capacidade de retenção de água no longo prazo, por meio de sua natureza frequentemente porosa, reflexo das estruturas celulares da matéria-prima. Também possui cinzas que são fontes de $\mathrm{P}, \mathrm{K}$ e outros elementos que podem estar em formas mais solúveis e acessíveis do que na matéria-prima não pirolisada. $\mathrm{O}$ efeito do biocarvão na disponibilidade destes elementos pode explicar alguns impactos na produção de algumas culturas a curto prazo, especialmente pelo fato de que alguns elementos não podem ser disponibilizados simplesmente pelo aumento da quantidade de matéria orgânica no solo (SOHI et al., 2010).

Em solos adubados anualmente com $4,5 \mathrm{t} \mathrm{ha}^{-1}$ de uma mistura de cinzas e biocarvão, este proveniente de usina termoelétrica, Omil et al. (2013) observaram que ao longo de três anos não foi necessária a aplicação suplementar de $\mathrm{P}$ para o crescimento de um povoamento de Pinus radiata com 13 anos de idade. Os autores ressaltaram que os tratamentos com a mistura reduziram perdas de nutrientes por lixiviação e aumentaram o teor de matéria orgânica do solo, mesmo em solo arenoso. Petter et al. (2012) afirmaram que a aplicação de biocarvão de eucalipto em aplicações de 8,16 e $32 \mathrm{t} \mathrm{ha}^{-1} \mathrm{em}$ campo, afetou positivamente a fertilidade do solo, aumentando principalmente a disponibilidade de Ca e $\mathrm{P}$, bem como aumentando o $\mathrm{pH}$ e o carbono orgânico total dos solos. Estudando a influência do biocarvão em duas doses, 8 ou 20 t ha $^{-1}$, aplicadas uma única vez, na qualidade do solo adubado, Major et al. (2010) observaram, após quatro anos da aplicação em savana colombiana, que o biocarvão, produzido a partir de resíduos de madeira, aumentou principalmente o pH e a disponibilidade de $\mathrm{Ca}$ e $\mathrm{Mg}$, e diminuiu a acidez trocável.

Zhao et al. (2014) concluíram que a aplicação de biocarvão de palha de arroz, em experimento em casa de vegetação, aumentou a produtividade de arroz e trigo e a absorção de $\mathrm{N}, \mathrm{P}, \mathrm{K}, \mathrm{Ca}$ e $\mathrm{Mg}$ pelas plantas, devido principalmente à disponibilidade de $\mathrm{P}, \mathrm{K}, \mathrm{Ca}$ e $\mathrm{Mg}$ dos solos incorporados com biocarvão. Já Alburquerque et al. (2013), trabalhando com biocarvões de palha de trigo e dos resíduos da poda de oliveiras, afirmaram que os biocarvões atuaram como fonte de $\mathrm{P}$ em solos após dois meses de incubação em casa de vegetação. Em contrapartida, a adição de qualquer um dos dois tipos de biocarvão resultou em perdas na disponibilidade de $\mathrm{N}$ e $\mathrm{Mn}$.

Novak et al. (2009) avaliaram a influência da adição de biocarvão de casca do fruto de nogueirapecã para a fertilidade do solo. Após 67 dias de incubação em casa de vegetação, o biocarvão aumentou o $\mathrm{pH}$ do solo, o carbono orgânico total e alguns nutrientes, e diminuiu a acidez trocável, $\mathrm{S}$ e Zn. Os autores concluíram que este biocarvão apresentou alta capacidade de sorção de nutrientes, principalmente $\mathrm{Ca}, \mathrm{P}$, $\mathrm{Zn}$ e Mn.

Em trabalho realizado com 17 biocarvões provenientes de diferentes matérias-primas e diferentes métodos de produção, Brewer et al. (2011) encontraram que, no geral, os biocarvões aumentaram o pH dos solos, após oito semanas de avaliação. Os biocarvões produzidos por gaseificação de resíduos de gramíneas proporcionaram as mais altas condutividades elétricas, que é um indicador do conteúdo de íons disponíveis no solo (BREWER et al., 2011).

Van Zwieten et al. (2010) observaram aumento de $\mathrm{pH}$, capacidade de troca de cátions, $\mathrm{K}, \mathrm{Ca}$ e carbono total após a aplicação de $10 \mathrm{t} \mathrm{ha}^{-1}$ de biocarvão de resíduo de indústria de celulose, em experimento em casa de vegetação. Ainda notaram redução da acidez trocável e aumento na absorção de $\mathrm{N}$ por plantas de trigo após aplicação do biocarvão.

Avaliando a capacidade potencial do biocarvão em melhorar a retenção de água em experimento em casa de vegetação, Devereux et al. (2012) constataram que o tamanho médio dos poros diminuiu de 0,07 $\mathrm{mm}^{2}$, no tratamento sem biocarvão, para $0,046 \mathrm{~mm}^{2}$, no tratamento com $5 \%$ de biocarvão no solo (v:v). Também foi observado que o aumento da concentração de biocarvão no solo diminuiu a condutividade hidráulica, densidade do solo e a repelência de água no solo. Os autores concluíram que o biocarvão pode

Ci. Fl., v. 28, n. 2, abr. - jun., 2018 
aumentar a retenção de água em solos propensos a períodos de seca. Já Liang et al. (2014) observaram que a aplicação de $90 \mathrm{t} \mathrm{ha}^{-1}$ de biocarvão aumentou o $\mathrm{pH}$ do solo e a capacidade de retenção de água, e reduziu a densidade do solo, depois de três anos da data de aplicação do biocarvão. Os autores também observaram que apesar da redução da disponibilidade de $\mathrm{N}$, a aplicação do biocarvão aumentou a disponibilidade de K.

Em trabalho de revisão de literatura, Warnock et al. (2007) demonstraram que a adição de diferentes tipos de biocarvão pode alterar a disponibilidade de nutrientes por modificar as propriedades físico-químicas do solo. Não menos importante, os autores desta revisão afirmam que o biocarvão apresenta efeito positivo para aumento da incidência de fungos micorrízicos (apenas poucos estudos apresentaram efeito negativo), mostrando que estes fungos respondem positivamente à aplicação de biocarvão. Os autores também descreveram quatro mecanismos de atuação do biocarvão para os fungos micorrízicos: o biocarvão aumenta a disponibilidade de nutrientes do meio, primordial para o crescimento dos fungos; o biocarvão altera a atividade de outros microrganismos que tem efeito nas micorrizas; o biocarvão modifica a dinâmica da rizosfera, aumentando a interação entre micróbios, raízes das plantas e fungos micorrízicos; e biocarvão atua como refúgio para fungos e bactérias colonizadores.

Também é observada a interação do biocarvão com o solo na adsorção de substâncias prejudiciais ao meio ambiente, normalmente presentes em herbicidas e pesticidas. Spokas et al. (2009) notaram que a adição de biocarvão, em quantidades superiores a $60 \mathrm{t} \mathrm{ha}^{-1}$, aumentou a adsorção de atrazina e acetocloro comparado ao solo-controle, sem adição de biocarvão, resultando na diminuição da lixiviação destas substâncias dos herbicidas avaliados. Zhang et al. (2012) avaliaram o efeito do biocarvão de Pinus radiata na adsorção de fenantreno, um hidrocarboneto aromático policíclico, prejudicial ao meio ambiente, que é formado a partir da combustão incompleta de matérias que contêm carbono, principalmente em lixões e refinarias de petróleo e xisto. Os autores observaram que o biocarvão produzido a $700^{\circ} \mathrm{C}$ foi mais efetivo para a adsorção da substância que o biocarvão produzido a $350^{\circ} \mathrm{C}$, devido à maior superfície específica do primeiro. Yu, Ying e Kookana (2009) notaram que solos tratados com mais de $5 \mathrm{t} \mathrm{ha}^{-1}$ de biocarvão de Eucalyptus spp. foram capazes de reduzir a absorção de clorpirifós e carbofurano por plantas de cebola (Allium серa), componentes químicos presentes em herbicidas e inseticidas que podem ser prejudiciais à saúde humana.

\section{BIOCARVÃO E PRODUTIVIDADE AGRÍCOLA}

$\mathrm{Na}$ agricultura, o uso do biocarvão tem resultados significativos nos ganhos em produtividade. Em uma revisão com a utilização de quase 50 trabalhos publicados, através de uma análise quantitativa dos efeitos da aplicação de biocarvão ao solo na produtividade das culturas utilizando "meta-análise", Jeffery et al. (2011) observaram que, no geral, a utilização do biocarvão aumentou em média a produtividade agrícola em $10 \%$. No entanto, a variabilidade do resultado de incremento de produtividade foi enorme, variando desde $-28 \%$ até $39 \%$. A utilização de biocarvão em solos ácidos ou com pH neutro, e em solos de textura média ou arenosa, também apresentou efeitos significativos e positivos para a produtividade agrícola. Os autores ainda afirmam que as mais altas produtividades foram obtidas quando foram utilizadas $100 \mathrm{t} \mathrm{ha}^{-1} \mathrm{de}^{-}$ biocarvão. Entre matérias-primas, os biocarvões de cama de aviário proporcionaram os maiores ganhos em produtividade, enquanto que os de lodo de esgoto tiveram as menores.

Em estudo avaliando a interação de três tipos de solo e quatro tipos de biocarvão provenientes de palha de trigo, cavacos de madeira e poda de vinhedo, no crescimento de mostarda (Sinapis alba L.), cevada (Hordeum vulgare L.) e trevo-vermelho (Trifolium pretense L.), Kloss et al. (2014) observaram que o crescimento de mostarda, no primeiro ciclo, e de cevada, no segundo ciclo, foram reduzidos com a utilização de biocarvão. Os autores notaram que apenas o biocarvão de cavacos de madeira foi capaz de promover ganhos na produtividade de cevada, comparado ao tratamento-controle.

Petter et al. (2012) avaliaram a interação de doses do fertilizante mineral NPK e doses de biocarvão de eucalipto sobre a produtividade e o desenvolvimento do arroz de terras altas. Após um ano de avaliação, o biocarvão foi o único fator que apresentou efeito significativo com a produtividade de arroz. Já no segundo ano, apesar de ser superado pela produtividade apresentada nos tratamentos com fertilização, o biocarvão se correlacionou significativamente com o fertilizante no crescimento das plantas e no acúmulo de massa

Ci. Fl., v. 28, n. 2, abr. - jun., 2018 
de matéria seca.

Chan et al. (2007) observaram a importância da utilização de fertilizante nitrogenado juntamente com a aplicação do biocarvão de resíduos agrícolas (poda, grama e algodão), em experimento em casa de vegetação. Os autores notaram que mesmo com alta quantidade de biocarvão (100 t ha $\left.{ }^{-1}\right)$, mas na ausência de fertilizante, a produtividade de rabanete (Raphanus sativus) não foi melhorada. No entanto, com a aplicação de $100 \mathrm{~kg} \mathrm{~N} \mathrm{ha}^{-1}$, associado a $100 \mathrm{t} \mathrm{ha}^{-1}$ de biocarvão, a produtividade em massa seca aumentou $266 \%$.

Apesar de ser menos expressiva, a utilização de biocarvão para o crescimento de espécies florestais tem sido testada. Em um experimento em casa de vegetação, Pluchon et al. (2014), avaliando o crescimento de mudas de quatro espécies florestais produzidas em solos adubados com nove tipos de biocarvão provenientes de coníferas e folhosas, notaram que, em geral, a adição de biocarvão apresentou efeito positivo ou neutro no crescimento das mudas. Os autores ainda notaram que os biocarvões provenientes de angiospermas aceleraram o crescimento das mudas em comparação aos biocarvões provenientes de gimnospermas; e ainda, mudas de angiospermas responderam melhor ao biocarvão que as mudas de gimnospermas.

O uso de biocarvão de resíduos agrícolas no substrato proporcionou melhoria na emergência das sementes de sete espécies arbóreas avaliadas na Zâmbia (CHIDUMAYO, 1994). O autor ainda notou que o crescimento das mudas de seis, das sete espécies avaliadas, foi maior quando o biocarvão foi utilizado como componente de substrato. Apenas para uma das sete espécies avaliadas, a utilização do tratamento-controle, apenas solo como substrato para o crescimento das mudas, o biocarvão proporcionou médias inferiores no crescimento.

A aplicação de 4,5 $\mathrm{t} \mathrm{ha}^{-1}$ de uma mistura de biocarvão e cinzas, foi avaliada em um povoamento de Pinus radiata, com 13 anos de idade, em dois tipos de solo, por Omil et al. (2013). Os autores observaram respostas no crescimento em altura, diâmetro e volume principalmente após dois anos de aplicação da mistura. Os autores concluíram que o crescimento foi mais evidente no sítio de qualidade mais baixa. Ainda, sugerem que o biocarvão pode ser uma alternativa para uma silvicultura intensiva em sítios poucos produtivos em diferentes estágios de estabelecimento do povoamento, com aplicação antes do fechamento das copas ou logo após o desbaste.

Santalla et al. (2011), em trabalho realizado na aplicação de biocarvão e cinzas em um povoamento de Pinus radiata com 25 anos de idade, observaram que a aplicação da mistura, após quatro anos da aplicação, proporcionou maiores incrementos de altura e diâmetro que o tratamento-controle. No entanto, os autores encontraram que o tratamento combinado com Ca $\left(493 \mathrm{~kg} \mathrm{de} \mathrm{Ca} \mathrm{ha}^{-1}\right)$ e $\mathrm{P}\left(65 \mathrm{~kg} \mathrm{P} \mathrm{ha}^{-1}\right)$ apresentou as maiores médias de altura e diâmetro, passados quatro anos após a aplicação, sendo estatisticamente superior ao tratamento-controle, mas não ao tratamento com biocarvão e cinzas.

Avaliando o crescimento de Pseudotsuga menziesii aos cinco anos de idade, Solla-Gullon et al. (2006) observaram que a aplicação de biocarvão de indústria de papel e celulose contribuiu para o crescimento em altura do povoamento em 5,4 e 18\% após o quarto ano da aplicação de 10 e $20 \mathrm{t}^{\text {ha }}{ }^{-1}$, respectivamente, quando comparados ao tratamento-controle.

\section{BIOCARVÃO E O SEU EFEITO NA EMISSÃO DE GASES DO EFEITO ESTUFA}

Atualmente, não existe um termo que defina bem o processo de sequestro de $\mathrm{C}$ pelo uso do biocarvão. $\mathrm{O}$ termo sequestro de $\mathrm{C}$ no solo tipicamente se refere ao $\mathrm{C}$ que está estocado no solo como resultado de uma troca no manejo que aumenta o retorno da biomassa no solo ou diminui a sua decomposição. No entanto, tal sequestro é frequentemente debatido por questões de tempo de permanência e perdas de C no solo. Por contraste, a pirólise transforma a biomassa e estabiliza o carbono no biocarvão em uma forma que é mais resistente à decomposição quando aplicado ao solo (GAUNT; COWIE, 2009).

O manejo convencional da biomassa, como a deposição em aterros, conduz a uma liberação de metano $\left(\mathrm{CH}_{4}\right)$ e óxido nitroso $\left(\mathrm{N}_{2} \mathrm{O}\right)$, gases que contribuem para o aumento do efeito estufa, já que a biomassa se decompõe sob condições anaeróbicas. Estratégias de manejo que evitem estas emissões podem contribuir significantemente para mitigar problemas ambientais. Muitos resíduos orgânicos são passives para a produção de biocarvão. A quantidade de emissões evitadas através da transformação em biocarvão é 
dependente da natureza da biomassa utilizada e do manejo envolvido. No processo de produção de biocarvão, a liberação inicial de gases de efeito estufa é mais rápida do que ocorreria durante a decomposição natural, contudo, a maioria da porção remanescente é provavelmente mais estável, por centenas de anos quando adicionada ao solo (GAUNT; COWIE, 2009).

Lehmann et al. (2006) estimam que até $12 \%$ do total das emissões de C por mudança no uso da terra poderiam ser evitadas anualmente desde que o sistema tradicional slash-and-burn (corte e queima) fosse substituído pelo então denominado slash-and-char (corte e produção de biocarvão).

Enquanto materiais lenhosos apresentam um conteúdo de $50 \%$ de $\mathrm{C}$, o biocarvão possui cerca de 70 a $80 \%$ de C, que podem ser permanentemente depositados no solo. O carbono lábil tem meia-vida de um a cinco anos, o carbono húmico, de décadas, e o carbono orgânico inerte, como o do biocarvão, pode durar milhares de anos. Para cada tonelada de $\mathrm{C}$ perdida no solo, adiciona-se 3,67 toneladas de $\mathrm{CO}_{2}$ na atmosfera (WINSLEY, 2007).

Lehmann (2007a) acredita que quando combinado com a produção bioenergética, ou seja, com a captação dos gases produzidos durante a pirólise, o biocarvão é uma tecnologia limpa para sequestro de carbono. E é, portanto, um alvo atrativo para subsídios à produção energética e para a sua inclusão no mercado global de créditos de carbono (Figura 1). O autor ainda afirma que $10 \%$ das emissões anuais de $\mathrm{C}$ dos Estados Unidos poderiam ser evitadas através da utilização do biocarvão.
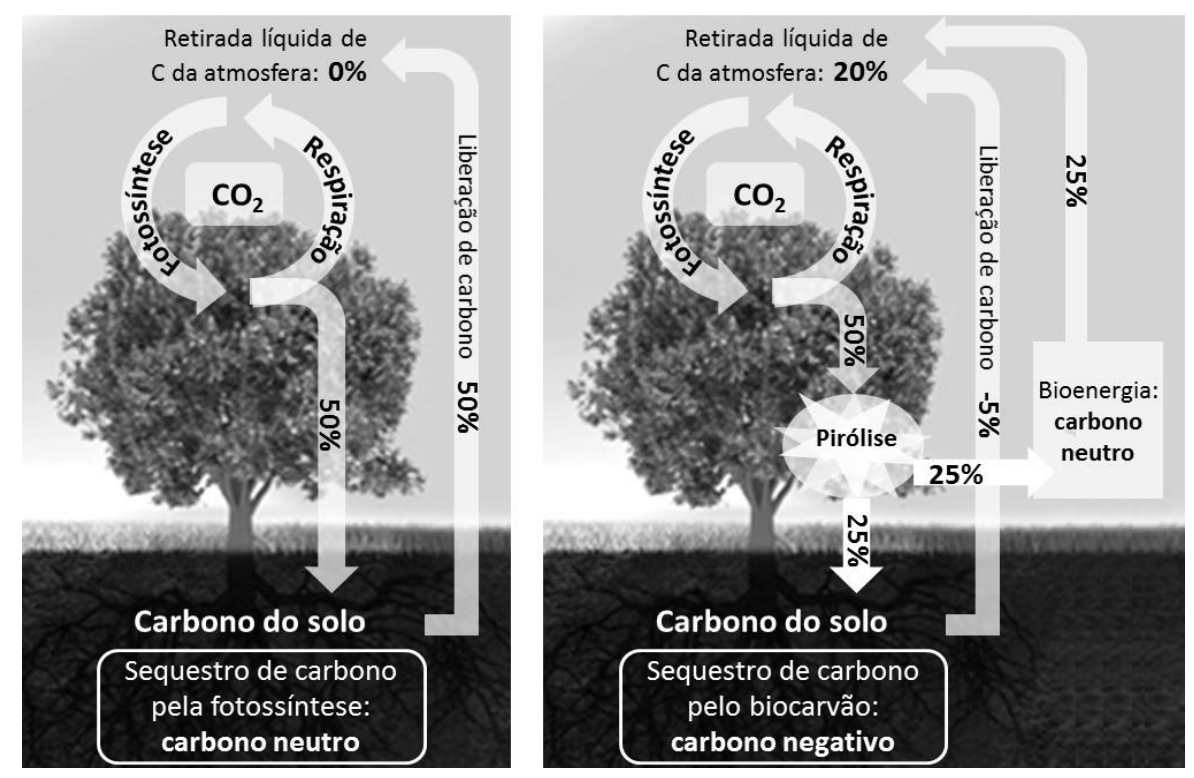

FIGURA 1: Ciclo do carbono e sequestro de carbono pelo uso do biocarvão (adaptado de LEHMANN, 2007a).

FIGURE 1: Carbon cycle and biochar carbon sequestration.

Muito pouco se sabe sobre tempo de meia-vida do biocarvão no solo, principalmente por duas razões: primeiramente, a recalcitrância do biocarvão depende de uma série de fatores, incluindo o tipo de biomassa usado na pirólise, das condições de produção, das propriedades do solo e das condições climáticas. Em segundo lugar, a quantificação da estabilidade requer observações em longo prazo, superior aos períodos viáveis de experimentos tradicionais (LEHMANN, 2007b).

Ghani et al. (2013) avaliaram o potencial do biocarvão produzido a partir de serragem de pinus no sequestro de $\mathrm{C}$, através de suas características físico-químicas. Os autores observaram que com o aumento da temperatura, o conteúdo de $\mathrm{C}$ também foi aumentado, mostrando um efeito inverso nas relações atômicas $\mathrm{H} / \mathrm{C}$ e $\mathrm{O} / \mathrm{C}$. Os resultados de infravermelho indicaram um grupo funcional aromático no comprimento de $866 \mathrm{~cm}^{-1} \mathrm{em}$ muitas amostras, mostrando recalcitrância no material produzido. Ainda concluíram que a taxa de adsorção de $\mathrm{CO}_{2}$ no biocarvão aumentou com o aumento da temperatura de pirólise de 450 a $650^{\circ} \mathrm{C}$, e então, ocorrendo posterior diminuição com o aumento da temperatura. 
Nguyen et al. (2008) estimaram o tempo de permanência do C em solos no Quênia, em oito diferentes momentos de coleta (de dois a 100 anos), em florestas que sofreram queimadas. Os autores observaram que o conteúdo de $\mathrm{C}$ decresceu rapidamente, de 12,7 a 3,8 $\mathrm{mg} \mathrm{C} \mathrm{g}^{-1}$ de solo, nos 30 primeiros anos de deposição. Depois disso houve uma pequena diminuição no conteúdo de carbono até atingir a estabilização em $3,5 \mathrm{mg}$ $\mathrm{C} \mathrm{g}^{-1}$ de solo. Os autores ainda estimaram que em 100 anos a perda total de $\mathrm{C}$ foi ao redor de $6000 \mathrm{~kg} \mathrm{C} \mathrm{ha}^{-1}$.

Para Ippolito et al.(2012), o biocarvão é um agente efetivo no sequestro de C nos solos, principalmente se produzido em moderadas ou altas temperaturas, pois se torna estável contra a ação decompositora de microrganismos e então persistirá por centenas ou até milhares de anos nos solos. No entanto, o impacto na rede de gases de efeito estufa é dependente da aplicação do biocarvão no solo no aumento da eficiência da mineralização ou humificação, na ciclagem de matéria orgânica do solo e nas emissões de $\mathrm{CH}_{4}$ e $\mathrm{N}_{2} \mathrm{O}$.

Em experimento em casa de vegetação, Zhao et al. (2014) avaliaram os efeitos da aplicação de biocarvão de palha de arroz em solo para o sequestro de $\mathrm{C}$ e emissão de gases de efeito estufa. Os autores

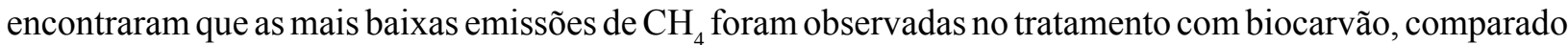
ao tratamento-controle e ao tratamento com palha de arroz in natura, mesmo o biocarvão trazendo mais $\mathrm{C}$ ao solo. Já as emissões de $\mathrm{N}_{2} \mathrm{O}$ foram similares entre o tratamento-controle e o tratamento com biocarvão.

Mukherjee et al. (2014) avaliaram a utilização de biocarvão (carvão comercial), águas residuais e ácido húmico, adicionados em solo argilo-siltoso, em experimento em campo. Depois de quatro meses de incubação, os autores não observaram diferenças significativas entre os tratamentos para as emissões acumuladas de $\mathrm{CH}_{4}$ e $\mathrm{CO}_{2}$, no entanto, o biocarvão reduziu em $92 \%$ as emissões de $\mathrm{N}_{2} \mathrm{O}$, comparado ao tratamento-controle. Os autores ainda ressaltaram que considerando o $\mathrm{C}$ removido pela atmosfera (através do crescimento da soja) e o C adicionado, o tratamento com biocarvão proporcionou um efeito de emissão negativa, em que a capacidade de armazenamento foi superior à liberação de $\mathrm{C}$.

Avaliando as emissões de gases de efeito estufa, em experimento em campo, Zhang et al. (2012) observaram reduções nas emissões $\mathrm{N}_{2} \mathrm{O}$ em 10,7 e 41,8\% nos tratamentos com 20 e 40 t ha- ${ }^{-1}$ de biocarvão de palha de trigo, respectivamente, comparados ao tratamento-controle. No entanto, o tratamento com a maior quantidade de biocarvão aplicada $\left(40\right.$ t ha $\left.^{-1}\right)$ aumentou em $12 \%$ as taxas de emissão de $\mathrm{CO}_{2}$, em comparação ao controle.

\section{CONTEXTUALIZAÇÃO POLÍTICA, RISCOS E INCERTEZAS SOBRE O BIOCARVÃO}

Estudos com o biocarvão já atingiram proporções de escala mundial que envolvem diversas áreas como Ciências Ambientais, Energia, Ciência do Solo, Biotecnologia e Microbiologia, Engenharia Química, Engenharia Ambiental e Agronomia (WEB OF SCIENCE, 2017). Sua pesquisa científica tem crescido em grandes proporções nos últimos anos, sendo que seu primeiro artigo (considerando o termo em inglês biochar) foi publicado em 2000, e atingiu um número superior a 1200 artigos publicados em 2016 (Figura 2). No entanto, ainda existem muitas incertezas sobre a sua utilização na agricultura, pois a maioria dos trabalhos publicados supervaloriza o potencial do biocarvão, dando menor preferência aos esclarecimentos dos riscos envolvidos do que a sua capacidade de manutenção e melhoria da fertilidade do solo e aumento da produtividade agrícola.

De acordo com Mukherjee e Lal (2014), os experimentos que avaliam o biocarvão como condicionador do solo apresentam escassez na quantidade de dados em nível de campo para respostas do crescimento vegetal, para a qualidade do solo e no impacto ambiental. Os autores ainda comentam que as respostas do biocarvão como condicionador de solo são baseadas em um curto período de tempo e em estudos de laboratório ou casa de vegetação, e por isso são muitas vezes inconclusivos e contraditórios.

Ainda, muito tem se falado sobre a inserção do biocarvão em programas de sequestro de carbono e de gases do efeito estufa. Para inserção do biocarvão em programas de crédito de carbono, deve-se considerar que o sistema seja verificável, transparente e real. Um protocolo de carbono deve refletir todos estes requisitos essenciais para a sua inserção. No entanto, o volume exato de créditos de carbono gerados por projetos de incorporação de biocarvão ao solo é determinado por um grande número de fatores relacionados com a fonte de matéria-prima, o método de produção, transporte utilizado, o procedimento de incorporação e características do local onde o biocarvão acabará por ser incorporado (DE GRYZE et al., 2010). A adição 


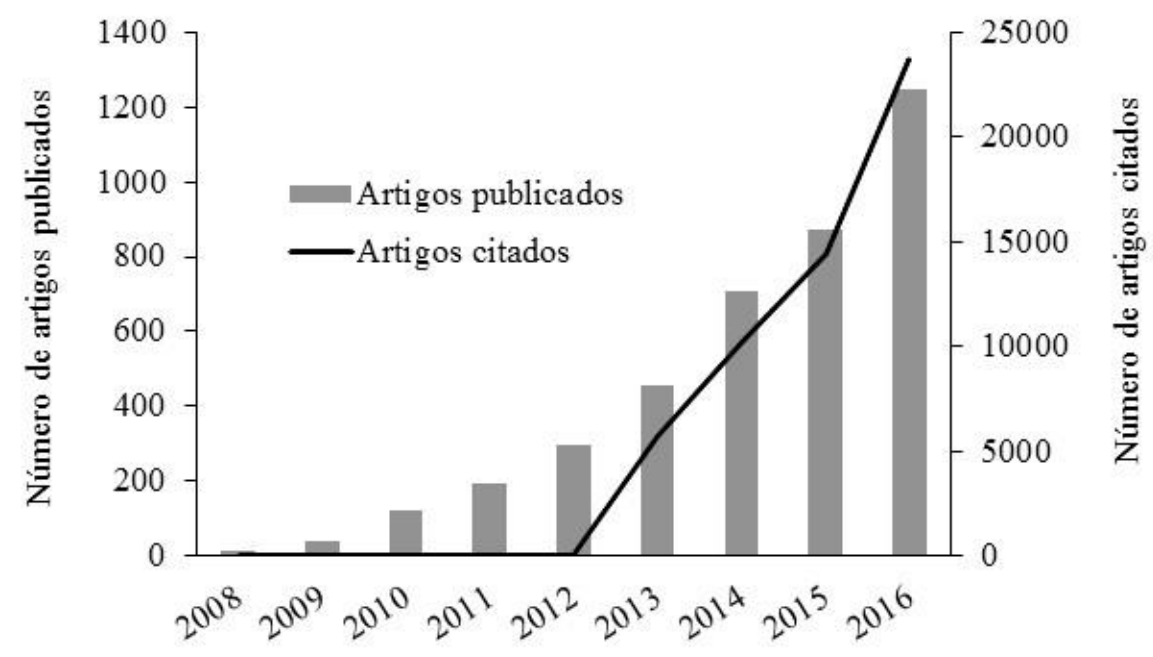

FIGURA 2: Artigos publicados sobre o tema biocarvão (biochar) nos últimos anos.

FIGURE 2: Biochar published papers in recent years.

de biocarvão poderia também promover uma rápida perda de húmus da floresta e carbono do solo em alguns ecossistemas durante as primeiras décadas (WARDLE; NILSSON; ZACKRISSON, 2008).

Ainda devem ser considerados os riscos iminentes envolvidos na produção, manuseio e utilização do biocarvão. As diferentes matérias-primas utilizadas, como biossólidos, águas residuais e estercos de animais podem conter patógenos que podem contaminar o solo, e ainda não se sabe a real influência da pirólise na resolução deste problema. A potencial presença de metais pesados e outros elementos perigosos nos biocarvões podem colocar em risco a sua utilização como condicionador de solo (BRACMORT, 2010; DE GRYZE et al., 2010).

No processo de produção de biocarvão, a pirólise também proporciona a formação de bio-óleos, que pode apresentar pequenos níveis de hidrocarbonetos aromáticos policíclicos (HAP) (SOHI et al., 2009). Os HAP são compostos que podem causar danos ambientais irreparáveis e são formados sempre que materiais formados por carbono são queimados parcialmente, tal como a produção do biocarvão. A possibilidade da presença de HAP no biocarvão pode ser um limitante para sua utilização como condicionador de solo (DE GRYZE et al., 2010). Garcia-Perez (2008) comenta que a queima de sólidos em faixas de temperatura de $350-600^{\circ} \mathrm{C}$ são produzidas pequenas quantidades de HAP, principalmente de baixo peso molecular, que apresentam menor risco. Porém, também podem ser produzidos HAP de maiores pesos moleculares, cujos riscos são maiores.

As partículas muito pequenas de biocarvão podem contaminar o ar e ainda aumentar os riscos de incêndio. Blackwell et al. (2009) afirma que as partículas menores que $10 \mu \mathrm{m}$ presentes no biocarvão são facilmente levadas por ventos leves durante a estocagem, transporte ou aplicação. Além da poluição atmosférica causada pela poeira, existe ainda o maior risco de incêndio causado pelas menores partículas de biocarvão (DE GRYZE et al., 2010).

Em se tratando do aspecto financeiro, a viabilidade econômica do biocarvão é depende do impacto da sua utilização para a produtividade da cultura, do custo de produção, matéria-prima e transporte para local de aplicação (BURNS et al., 2014). Os autores consideram ainda que os projetos com o uso do biocarvão serão mais rentáveis quando existir um mercado de carbono mais consolidado (BURNS et al., 2014). Novotny et al. (2015) sugerem uma avaliação econômica do uso do biocarvão como condicionador de solo como um substituto ao calcário. Blackwell et al. (2010) afirmam que o custo de aplicação e incorporação de biocarvão no solo será rentável até um valor de $\$ 170 /$ ha, proporcionando um aumento de produtividade em torno de $10 \%$ durante doze anos.

Ainda vale ressaltar os diversos interesses proporcionados pela possibilidade de obtenção de lucro pela produção e utilização do biocarvão. Devido a diversas pesquisas ligarem o biocarvão à "Terra Preta de Índio" (TPI), existe um interesse comercial de grandes empresas que utilizam de lobistas que promovem o biocarvão por declararem a sua similaridade com as TPI (ERNSTING; SMOLKER, 2009; ERNSTING, 
2011). Os autores acreditam que a capacidade de dreno de $C$ pelo biocarvão tem sido superestimada principalmente pelo fato de que as pesquisas não têm considerado os numerosos impactos sociais e ambientais.

Por interesse particular ou não, o biocarvão foi citado no quinto relatório do IPCC (Intergovernmental Panel on Climate Change - Painel Intergovernamental sobre Mudanças Climáticas) como uma potencial nova tecnologia que poderia contribuir para a mitigação de gases do efeito estufa incluídos no setor de Agricultura, Floresta e Uso do Solo, contudo, ainda são limitadas as evidências que fazem estimativas robustas que comprovem a sua real eficácia (INTERGOVERNMENTAL PANEL ON CLIMATE CHANGE, 2014).

Em escala nacional, o biocarvão tem sido objeto de estudo de algumas instituições públicas. No entanto, tratando-se sobre a possibilidade de mitigação da emissão de gases de efeito estufa, o biocarvão ainda não foi incluso no Plano ABC (Agricultura de Baixa Emissão de Carbono) do Governo Federal (BRASIL, 2012).

\section{CONSIDERAÇÕES FINAIS}

A utilização de biocarvão no meio florestal apresenta um grande potencial a ser explorado, principalmente na silvicultura, como condicionador de solo ou substrato para produção de mudas. Para a sua real efetivação torna-se necessário o aumento das pesquisas no meio florestal, considerando principalmente uma maior e mais detalhada caracterização físico-química do biocarvão utilizado, bem como nos processos de produção, procurando preencher todas as lacunas da real contribuição do biocarvão na interação com o solo e no crescimento de espécies florestais. Estudos com períodos mais longos de observações e com sucessivos ciclos de crescimento são requeridos para que o biocarvão possa ser recomendado em escala comercial e do ponto de vista prático.

\section{AGRADECIMENTOS}

O primeiro autor agradece ao Conselho Nacional de Desenvolvimento Científico e Tecnológico (CNPq) pela concessão da bolsa de Pós-doutorado Júnior e Pós-doutorado no Exterior.

\section{REFERÊNCIAS}

ALBURQUERQUE, J. A. et al. Enhanced wheat yield by biochar addition under different mineral fertilization levels. Agronomy for Sustainable Development, Paris, v. 33, p. 475-484, 2013.

ATKINSON, C. J. et al. Potential mechanisms for achieving agricultural benefits from biochar application to temperate soils: a review. Plant and Soil, Amsterdam, v. 337, p. 1-18, 2010.

BLACKWELL, P. et al. Biochar application to soil. In: LEHMANN, J.; JOSEPH, S. (Ed.). Biochar for environmental management: science and technology. London: Earthscan, 2009. p. 207-226.

BLACKWELL, P. et al. Effect of banded biochar on dryland wheat production and fertiliser use in southwestern Australia: an agronomic and economic perspective. Australian Journal of Soil Research, Canberra, v. 48, p. 531-545, 2010.

BRACMORT, K. Biochar: examination of an emerging concept to mitigate climate change. [s. 1.]: Congressional Research Service Report, 2010.

BRASIL. Ministério da Agricultura, Pecuária e Abastecimento. Plano setorial de mitigação e de adaptação às mudanças climáticas para a consolidação de uma economia de baixa emissão de carbono na agricultura: plano ABC (Agricultura de Baixa Emissão de Carbono). Brasília: MAPA/ACS, 2012. 173 p. disponível em:

$<$ http://www.agricultura.gov.br/arq_editor/download.pdf $>$. Acesso em: 10 out. 2014.

BREWER, C. E. et al. Criteria to select biochars for field studies based on biochar chemical properties.

Bioenergy Research, New York, v. 4, p. 312-323, 2011.

BROWN, R. Biochar production technology. In: LEHMANN, J.; JOSEPH, S. (Ed.). Biochar for 
environmental management: science and technology. London: Earthscan, 2009. p. 127-148.

BROWNSORT, P. A. Biomass pyrolisis processes: review of scope, control and variability. United Kingdom Biochar Research Centre, London, v. 1, p. 1-39, 2009.

BURNS, C. et al. The economics of biochar production: a review. Nevada: University of Nevada, 2014. CHAN, K. Y. et al. Agronomic values of greenwaste biochar as a soil amendment. Australian Journal of Soil Research. Canberra, v. 45, p. 629-634, 2007.

CHIDUMAYO, E. N. Effects of wood carbonization on soil and initial development of seedlings in miombo woodland, Zambia. Forest Ecology and Management, Amsterdam, v. 70, p. 353-357, 1994.

DE GRYZE, S. et al. Evaluation of the opportunities for generating carbon offsets from soil sequestration of biochar. San Francisco: Terra Global Capital, 2010.

DEVEREUX, R. C. et al. The effects of biochar on soil physical properties and winter wheat growth. Earth and Environmental Science Transactions of the Royal Society of Edinburgh, Edinburgh, v. 103, p. 13-18, 2012.

DOWNIE, A. et al. Physical Properties of Biochar. In: LEHMANN, J.; JOSEPH, S. Biochar for environmental management: science and Technology. London: Earthscan, 2009. p. 13-32.

ERNSTING, A. Biochar - a climate smart solution? Bischöfl iches Hilfswerk MISEREOR, Aachen, v. 1, p. 1-24, 2011.

ERNSTING, A.; SMOLKER, R. Biochar for climate change mitigation: fact or fiction? 2009. 10 p. Disponível em: <www.biofuelwatch.org.uk/docs/biocharbriefing.pdf > Acesso em: 10 out. 2014.

GARCIA-PEREZ, M. The Formation of polyaromatic hydrocarbons and dioxins during pyrolysis. Washington: Washington State University, 2008. 63 p.

GAUNT, J.; COWIE A. Biochar, greenhouse gas accounting and emissions trading. In: LEHMANN, J.; JOSEPH, S. (Ed.). Biochar for environmental management: science and Technology. London: Earthscan, 2009. p. 317-340.

GHANI, W. A. W. A. K. et al. Biochar production from waste rubber-wood-sawdust and its potential use in $\mathrm{C}$ sequestration: chemical and physical characterization. Industrial Crops and Products, Amsterdam, v. 44, p. 18-24, 2013.

GLASER, B. et al. The "Terra Preta" Phenomenon: a model for sustainable agriculture in the humid tropics'. Naturwissenschaften, Berlin, v. 88, p. 37-41, 2001.

INTERGOVERNMENTAL PANEL ON CLIMATE CHANGE. Summary for Policymakers. In: INTERGOVERNMENTAL PANEL ON CLIMATE CHANGE. Climate Change 2014: mitigation of climate change. Cambridge: Cambridge University Press, 2014. 2108 p. Disponível em: $<$ http://www.ipcc. ch/report/ar5/wg3/>. Acesso em: 10 out. 2014.

IPPOLITO, J. A. et al. Environmental benefits of biochar. Journal of Environmental Quality, Madison, v. 41, p. 967-972, 2012.

JEFFERY, S. et al. A quantitative review of the effects of biochar application to soils on crop productivity using meta-analysis. Agriculture, Ecosystems and Environment, Zurich, v. 144, p. 175-187, 2011.

JOSEPH, S. et al. An investigation into the reactions of biochar in soil. Australian Journal of Soil Research, Canberra, v. 48, p. 501-515, 2010.

KLOSS, S. et al. Biochar application to temperate soils: effects on soil fertility and crop growth under greenhouse conditions. Journal of Plant Nutrition and Soil Science, Berlin, v. 177, p. 3-15, 2014.

KOOKANA, R. S. et al. Biochar application to soil: agronomic and environment benefits and unintended consequences. Advances in Agronomy, Madison, n. 112, p. 103-143, 2011.

LEHMANN, J. A handful of carbon. Nature, London, v. 447, p. 143-144, 2007 a.

LEHMANN, J. Bio-energy in the black. Frontiers in Ecology and the Environment, New York, v. 5, p. 381-387, 2007b.

LEHMANN, J. et al. Biochar effects on soil biota - A review. Soil Biology \& Biochemistry, London, v. 43, p. 1812-1836, 2011.

LEHMANN, J. et al. Biochar sequestration in terrestrial ecosystems - a review. Mitigation and Adaptation Strategies for Global Change, Berlin, v. 11. p. 395-419, 2006.

LEHMANN, J.; JOSEPH, S. Biochar for Environmental Management: an Introduction. In: LEHMANN, J.; JOSEPH, S. (Ed.). Biochar for environmental management: science and Technology. London: 
Earthscan, 2009. p. 1-12.

LIANG, B. et al. Black carbon increases cation exchange capacity in soils. Soil Science Society of American Journal, Madison, v. 70, p. 1719-1730, 2006.

LIANG, F. et al. Crop yield and soil properties in the first 3 years after biochar application to a calcareous soil. Journal of Integrative Agriculture, Beijing, v. 13, p. 525-532, 2014.

MAJOR, J. et al. Maize yield and nutrition during 4 years after biochar application to a Colombian savanna Oxisol. Plant and Soil, Amsterdam, v. 333, p. 117-128, 2010.

MCCARL, B. A. et al. Economics of biochar production, utilization and gas offsets. In: LEHMANN, J.; JOSEPH, S. (Ed). Biochar for Environmental Management: science and Technology. London: Earthscan, 2009. p. 341-356.

MUKHERJEE, A. et al. Effects of biochar and other amendments on the physical properties and greenhouse gas emissions of an artificially degraded soil. Science of the Total Environment, Barcelona, v. 487, p. 26-36, 2014.

MUKHERJEE, A.; LAL, R. The biochar dilemma. Soil Research, Camberra, v. 52, p. 217-230, 2014.

NGUYEN, B. T. et al. Long-term black carbon dynamics in cultivated soil. Biogeochemistry, New York, v. 89, p. 295-308, 2008.

NOVAK, J. M. et al. Designing relevant biochars as soil amendments using lignocellulosic-based and manure-based feedstocks. Journal of Soils and Sediments, Berlin, v. 14, p. 330-343, 2014.

NOVAK, J. M. et al. Impact of biochar amendment on fertility of a southeastern coastal plain soil. Soil Science, Berlin, v. 174, p. 105-112, 2009.

NOVOTNY, E. et al. Biochar: pyrogenic carbon for agriculture use - a critical review. Revista Brasileira de Ciência do Solo, Piracicaba, v. 39, p. 321-344, 2015.

NOVOTNY, E. et al. Lessons from the Terra Preta de Índios of the Amazon Region for the utilization of charcoal for soil amendment. Journal of the Brazilian Chemical Society, São Paulo, v. 20, n. 6, p. 1003-1010, 2009.

OMIL, B. et al. Soil and tree responses to the application of wood ash containing charcoal in two soils with contrasting properties. Forest Ecology and Management, Amsterdam, v. 295, p. 199-212, 2013.

PETTER, F. A. et al. Soil fertility and upland rice yield after biochar application in the Cerrado. Pesquisa Agropecuária Brasileira, Brasília, v. 47, n. 5, p. 699-706, 2012.

PLUCHON, N. et al. Stimulation of boreal tree seedling growth by wood-derived charcoal: effects of charcoal properties, seedling species and soil fertility. Functional Ecology, London, v. 28, p. 766-775, 2014.

RONDON, M. A. et al. Biological nitrogen fixation by common beans (Phaseolus vulgaris L.) increases with bio-char additions. Biology Fertility Soils, Amsterdam, v. 43, p. 699-708, 2007.

SANTALLA, M. et al. Effectiveness of wood ash containing charcoal as a fertilizer for a forest plantation in a temperate region. Plant and Soil, Amsterdam, v. 346, p. 63-78, 2011.

SOHI, S. P. et al. Biochar, climate change and soil: a review to guide future research. Camberra: CSIRO Land and Water Science Report, 2009. 64 p.

SOHI, S. P. et al. Review of biochar and its use and function in soil. In: SPARKS, D. L. (Ed.). Advances in Agronomy. Burlington: Academic Press, 2010. p. 47-82.

SOLLA-GULLON, F. et al. Nutritional status and growth of a young Pseudotsuga menziesii plantation in a temperate region after application of wood-bark ash. Forest Ecology and Management, Amsterdam, v. 237, p. 312-321, 2006.

SPOKAS, K. A. et al. Impacts of woodchip biochar additions on greenhouse gas production and sorption/ degradation of two herbicides in a Minnesota soil. Chemosphere, Amsterdam, v. 77, p. 574-658, 2009.

THIES, J. E.; RILLIG, M. C. Characteristics of Biochar: biological Properties. In: LEHMANN, J.; JOSEPH, S. (Ed.). Biochar for environmental management: science and Technology. London: Earthscan, 2009. p. 85-106.

VAN ZWIETEN, L. et al. Effects of biochar from slow pyrolysis of papermill waste on agronomic performance and soil fertility. Plant and Soil, Amsterdam, v. 327, p. 235-246, 2010.

VERHEIJEN, F. G. A. et al. Biochar application to soils: a critical scientific review of effects on soil properties, processes and functions. Luxembourg: EUR 24099 EN, Office for the Official Publications of 
the European Communities, 2009. 149 p.

WARDLE, D. A.; NILSSON, M. C.; ZACKRISSON, O. Fire-derived charcoal causes loss of forest humus. Science, New York, v. 320, p. 629, 2008.

WARNOCK, D. D. et al. Mycorrhizal responses to biochar in soil - concepts and mechanisms. Plant and Soil, Amsterdam, v. 300, p. 9-20, 2007.

WEB OF SCIENCE. “Biochar". [2017]. Disponível em: <webofknowledge.com>. Acesso em: 17 mar. 2017.

WINSLEY, P. Biochar and bioenergy production for climate change mitigation. New Zealand Science Review, Wellington, v. 64, p. 5-10, 2007.

YU, X. Y.; YING, G. G.; KOOKANA, R. S. Reduced plant uptake of pesticides with biochar additions to soil. Chemosphere, Amsterdam, v. 76, p. 665-671, 2009.

ZHANG, A. et al. Effect of biochar amendment on maize yield and greenhouse gas emissions from a soil organic carbon poor calcareous loamy soil from Central China Plain. Plant and Soil, Amsterdam, v. 351, p. 263-275, 2012.

ZHAO, X. et al. Successive straw biochar application as a strategy to sequester carbon and improve fertility: A pot experiment with two rice/wheat rotations in paddy soil. Plant and Soil, Amsterdam, v. 378, p. 279-294, 2014. 